\title{
Mesomorphic Structures of Protonated Surfactant-Encapsulated Polyoxometalate Complexes
}

\author{
Shengyan Yin, Wen Li, Jinfeng Wang, Lixin Wu*
}

State Key Laboratory of Supramolecular Structure and Materials, Jilin University, Changchun 130012, P. R. China

To whom correspondence should be addressed. E-mail: wulx@jlu.edu.cn

\section{Supporting Information}

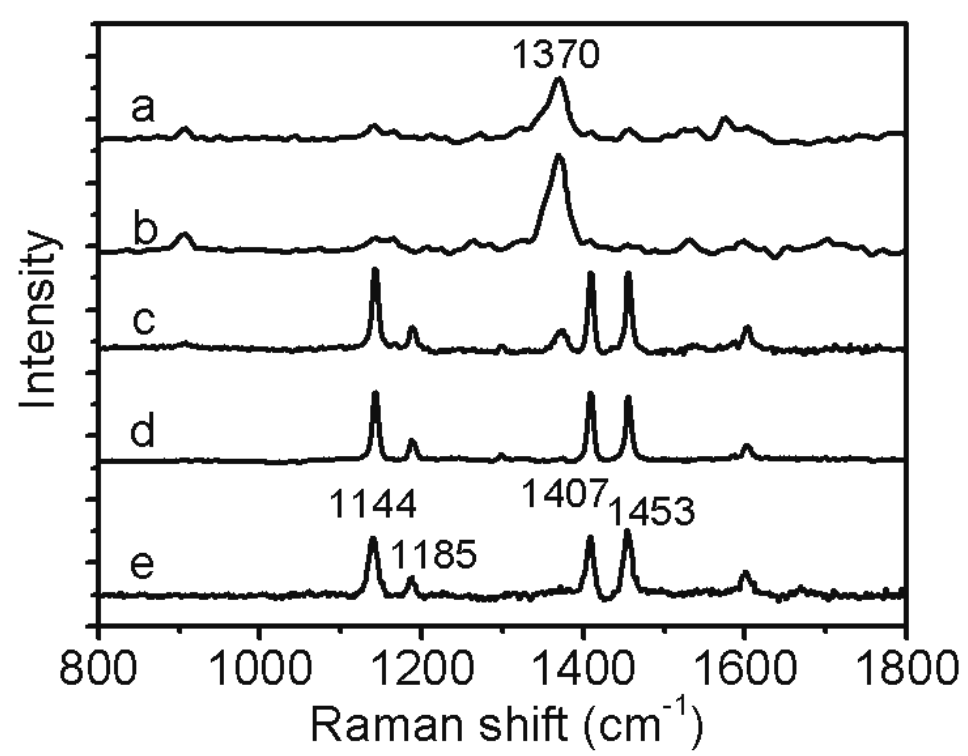

Figure S1. Raman spectra of the complexes, (a) HL/HPW, (b) HL/NaPW, (c) HL/HSiW, (d) L/KSiW and (e) L/HPW, excited by 514.5-nm line of Ar+ laser. 
TABLE 1: The Assignments of Characteristic Raman Shift in the Figure S1

\begin{tabular}{|c|c|}
\hline Raman shift $\left(\mathrm{cm}^{-1}\right)$ & Assignments \\
\hline 1144 & $-\mathrm{C}-\mathrm{N}=$ symmetric stretching \\
\hline 1185 & $-\mathrm{C}-\mathrm{N}=$ asymmetric stretching \\
\hline 1370 & protonated azobenzene stretching mode \\
\hline 1407 & $-\mathrm{N}=\mathrm{N}$ - trans- stretching modes \\
\hline 1453 & $-\mathrm{N}=\mathrm{N}$ - trans- stretching modes \\
\hline
\end{tabular}

\section{References}

[1] (a) Wu, Y.; Zhao, B.; Xu, W.; Li, B.; Jung, Y. M.; Ozaki, Y. Langmuir 1999, 15, 4625. (b) Tecklenburg, M. M. J.; Kosnak, D. J.; Bhatnagar, A.; Mohanty, D. K. J. Raman Spectrosc. 1997, 755. (c) Takahashi, H.; Umemura, J.; Takenaka, T. J. Phys. Chem. 1982, 86, 4660. 